\title{
Supply chain performance evaluation using robust data envelopment analysis
}

\author{
Alireza Arshadi Khamseh ${ }^{\mathrm{a}^{*}}$ and Dariush Zahmatkesh ${ }^{\mathrm{b}}$
}

${ }^{a}$ Department of Industrial Engineering, Faculty of Engineering, Kharazmi University, Tehran ,Iran ${ }^{b}$ Department of Industrial Engineering Kharazmi University, Tehran, Iran

\begin{tabular}{l}
\hline C H R O N I C L E \\
\hline Article history: \\
Received July 18, 2014 \\
Accepted January 172015 \\
Available online \\
January 172015 \\
\hline Keywords: \\
Robust modeling \\
Supply chain Management (SCM) \\
Data Envelopment Analysis \\
(DEA) \\
Linear programming (LP) \\
Performance Measurement \\
\end{tabular}
\begin{abstract}
A B S T R A C T
In this paper, we evaluate the performance of a supply chains (SCs) under uncertainty with different components such as direct costs, operational costs, transaction expenses, order lead time, product flexibility and net profit. Data Envelopment Analysis (DEA) can be used for measuring the performance of supply chain problems. On the other hand, robust optimization approach is a powerful technique for handling problems faced with various environmental uncertainties. This paper combines these two concepts and proposes a method to evaluate SCs performance. The results of the proposed method, under any different environmental situation, show which ranking of SC's performance is better in a network. The preliminary results of the implementation of a real-world case study indicates that the method could be successfully used for performance measurement.
\end{abstract}

\section{Introduction}

Data uncertainty is presented for many real-world optimization problems. For example, in supply chain optimization, the actual demand for products, financial returns, actual material requirements and other resources are not precisely known when critical decisions need to be made. In engineering and science, data is subjected to measurement errors, which also constitute sources of data uncertainty in the optimization model. Robust Optimization (RO) is a modeling methodology, combined with computational tools to process optimization problems in which the data are uncertain and is only known to belong to some uncertainty set. Concepts of robustness and robust design optimization have been developed independently in different scientific disciplines, mainly in the fields of operations research and engineering design (Ben-Tal \& Nemirovski, 1998, 2000) and El-Ghaoui et al. (1998) put a significant step forward in RO theory by proposing models for uncertain linear problems with ellipsoidal uncertainties and solving the counterparts of the nominal problem in the form of conic quadratic problems. Supply Chain Management which appeared in the early 1990s, now playing an important role as competitive advantages between firms and global markets in uncertain business environment. Supply chain management contains planning and managing production/manufacturing,

* Corresponding author Tel: +98-9123188815

E-mail address: ar_arshadi@khu.ac.ir (A. Arshadi Khamseh)

(C) 2015 Growing Science Ltd. All rights reserved.

doi: 10.5267/j.uscm.2015.2.001 
transportation and distribution products from the first stage of process in preparing and delivering raw materials to plants up to delivery of finished products to customers, so SCM wants to do this duty with lower cost and higher efficiency in uncertain environments regarding to its elements; facilities, suppliers, customers, products and methods of inventory control, purchasing and distribution and their connections in an integrated network; that could be close or open loop. Open loop network in SCM has been started from material suppliers and finished by delivering to final customers, but in closed loop supply chain (Fig.1), products move from manufacturers to customers, and also there is another flow that leads defected products from customers to manufacturers to repair and move back to customers. Regarding these conditions and definitions and complexities, supply chain performance evaluation is an important fact in improving performance of supply chains. Indeed, the term performance evaluation is defined for analyzing and computing the measure of efficiency and effectiveness. As a result, the purpose of supply chain efficiency is to evaluate how well we use resources along all sections of supply chain to get the best result for business.

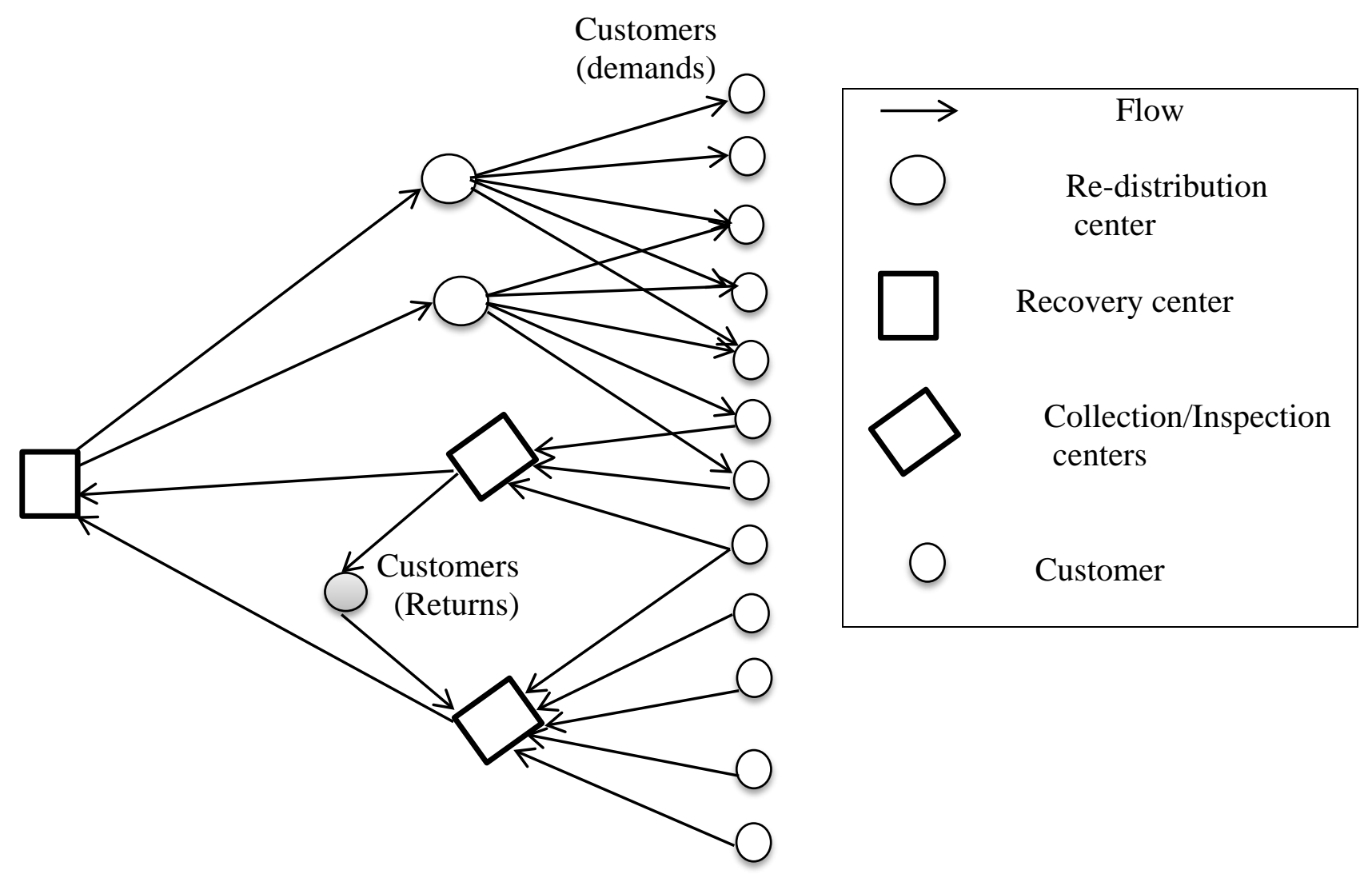

Fig. 1. A close loop supply chain.

In this paper, we want to evaluate the performance of an integrated supply chain with a Robust data envelopment analysis (DEA) model whereas input and output parameters of this model coming from uncertain environment. In part 2 we have a review of works which have been implemented on supply chain performance evaluation topic under certain or uncertain conditions, a brief introduction to robust modeling will be coming in part 3 and in section 4, transforming a linear CCR DEA model to a Robust Data Envelopment Analysis model will be defined, in section 5 these models (RDEA and classic DEA) will be applied to study supply chains performance evaluation in oil supporting industry via nominal and real data. The nominal and real data are addressed and they are taken from the oil supporting industry. In section 6 there is a comparison between two models and in section 7 conclusion will be presented. 


\section{Literature review}

During the past decade, most studies have been executed on evaluating the performance of specific parts of supply chain. Kleinsorge (1992) applied DEA methodology to evaluate the performance of different organizations among the supply chain. Chow et al. (1994) proposed the definition and measurement method on logistics performance problem. Barbarosoglu and Yazgac (1997), SinuanyStern et al. (2000) and Chan (2003) proposed methods like: Weighted linear methods, Linear programming, Analytical Hierarchy Process (AHP), Human Judgment Models, Data Envelopment Analysis (DEA), and Balanced Scored Cards (BSC). Ross and Droge (2002) evaluated the performance of distribution centers in a supply chain. Easton et al. (2002) evaluated the purchasing section of a supply chain and Talluria et al. (2006) evaluated the performance of different suppliers. Wu and Olson (2007) proposed a stochastic DEA model and compared it with a Multiple-criteria model in vendor selection problem. Wu et al. (2009) used stochastic DEA and Fuzzy DEA to evaluate performance of supply chain. In fact, these individual sections of a supply chain usually have different and often opposite goals, so we will use an integrated framework to evaluate the performance of all parts of supply chain together. During the recent years, robust optimization approach has been applied in some application areas such as inventory management and portfolio selection by researchers (e.g. Adida \& Perakis, 2006; El-Ghaoui et al., 1998; Gabrel et al., 2012; Amin \& Zhang, 2012). Sadjadi et al. (2011) presented a new method, which incorporates the robust counterpart of super-efficiency DEA. The perturbation and uncertainty in data is assumed as ellipsoidal set and the robust super-efficiency DEA model is extended. Chiang and Che (2010) applied the fuzzy AHP and fuzzy DEA to develop an evaluation and ranking methodology, assisting decision makers to select NPD projects with development potential and high added value which helps a company determine the direction of NPD for the future.

Khezrimotlagh et al. (2013) suggested a robust mixed integer linear programming based on the model developed earlier by Kourosh and Arash Method (KAM). The proposed linear model, integer-KAM (IKAM), has almost all capabilities of the linear KAM and significantly removes the shortcomings in the current MILPs. Mahmoudzadeh et al. (2013) developed a dynamic production/pricing problem, in which decisions should be made in each period confronting with uncertain demand and return. Sadjadi and Omrani (2008) proposed a DEA model with uncertain data for performance assessment of electricity distribution companies. Sadjadi and Omrani (2010) presented a robust data envelopment analysis (BRDEA) model for measuring the efficiency of telecommunication companies. Sadjadi et al. (2011) presented an interactive robust data envelopment analysis (IRDEA) model to determine the input and output target values of electricity distribution companies with considering the existence perturbation in data. Sadjadi et al. (2012) also showed a new portfolio modeling approach with uncertain data, which also used different robust optimization techniques. Finally the proposed model have been solved using genetic algorithm.

Sadjadi et al. (2014) proposed a capacitated multi-echelon, multi-product reverse logistic network design with fuzzy returned products in which both locations of the treatment activities and facilities were decision variables. Sadjadi et al. (2012) considered the project critical path problem in an environment with hybrid uncertainty. In this environment, the duration of activities were considered as random fuzzy variables that have probability and fuzzy natures, simultaneously. To obtain a robust critical path with this kind of uncertainty a chance constraints programming model was used. Soltani and Sadjadi (2010) proposed two hybrid meta-heuristics-hybrid simulated annealing and hybrid variable neighborhood search - to solve cross-docking system by achieving the best sequence of truck pairs.Because cross-docking system problem is NP-hard. 


\section{Robust optimization approach for uncertain data}

In this section uncertainty construction and its robust approach will be discussed:

\section{Uncertainty construction:}

Consider the following problem subject to data uncertainty:

$\min \mathbf{c x}$

subject to

$$
\begin{aligned}
& A x \leq b \\
& L \leq x \leq u
\end{aligned}
$$

In model (1) $\mathbf{c}$ is coefficient vector of $\boldsymbol{x}, \boldsymbol{x}$ is the vector of decision variables. We assume without any loss of generality that data uncertainty only affects the elements in matrix $\boldsymbol{A}$, then we model data uncertainty in $\boldsymbol{A}$ as follows:

Each uncertain coefficient $a_{i j}$ belongs to an interval, centered at its nominal value $\bar{a}_{i j}$ and half length $\hat{a}_{i j}$, but its exact value is unknown. It is unusual to assume that all coefficients are equal to their worstcase value; the worst-case value for all parameters; leads to the worst amount of cost. Hence, we wish to adjust the uncertainty level of the solution, so a reasonable trade-off between robustness, performance and costs will be achieved. To quantify this concept in mathematical terms, we define the scaled deviation of parameter $a_{i j}$ from its nominal value as $z_{i j}=\left(a_{i j}-\bar{a}_{i j}\right) / \hat{a}_{i j}$. The scaled deviation takes a value in interval $[-1,1]$. Moreover, we impose budget of uncertainty such as: The total (scaled) variation of the parameters cannot exceed threshold $\Gamma$, (not necessarily integer): $\quad \sum_{(i, j) \epsilon J}\left|z_{i j}\right| \leq \Gamma$ where $J$ is the set of indices of uncertain parameters. By taking $\Gamma=0(\Gamma=|J|)$ we obtain the nominal (worst) case. Sim (2004) showed that varying the threshold $\Gamma$ in $(0,|J|)$ allows greater flexibility to build a robust model without affecting the optimal cost.

The robust approach

Let $\Lambda=\left\{\begin{array}{c}\mathrm{A} \in \mathrm{R} \mid a_{i j} \in\left[\bar{a}_{\mathrm{ij}}-\hat{a}_{\mathrm{ij}}, \bar{a}_{\mathrm{ij}}+\hat{a}_{\mathrm{ij}}\right] \forall \mathrm{i}, \mathrm{j} \\ \sum_{(i, j) \epsilon J} \frac{\left|a_{i j}-\bar{a}_{\mathrm{ij}}\right|}{\hat{a}_{\mathrm{ij}}} \leq \Gamma\end{array}\right\}$. The robust problem is then formulated as follows,

$\min \boldsymbol{c x}$

subject to

$A x \leq b \quad \forall A \in \Lambda$

$L \leq x \leq u$

Theorem 1 (Ben Tal, 1998): uncertain linear programming problem (2) has the following robust linear counterpart:

$\min \boldsymbol{c} \boldsymbol{x}$

subject to

$\sum_{j} \bar{a}_{i j} x_{j}+q_{i} \Gamma+\sum_{j:(i, j) \epsilon J} r_{i j} \leq b_{i} \quad \forall i$

$q_{i}+r_{i j} \geq \hat{a}_{i j} y_{j} \quad \forall(i, j) \in J$

$-y \leq x \leq y \quad l \leq x \leq u$

$q \geq 0, r \geq 0, y \geq 0$.

Proof: [Sim]. 
The above robust counterpart has the same class as the nominal problem, that is, a linear programming , also if in the original problem (2), some of the variables have been constrained to be integers, then the robust counterpart (3) will have the same properties, i.e., the robust counterpart of a mixed integer programming problem will be a mixed integer too.

\section{Notation:}

Indices

I input index of each SC

$R \quad$ output index of each SC

$J \quad$ SC (Supply Chain) index (DMU)

$$
\begin{aligned}
& i=1,2,3,4 . \\
& r=1,2,3 . \\
& j=1, \ldots, 10 .
\end{aligned}
$$

Parameters

$X_{i j} \quad$ value of input $\mathrm{i}$ for $\mathrm{SC} \mathrm{j}$

$Y_{r j} \quad$ value of output $\mathrm{r}$ for $\mathrm{SC} \mathrm{j}$

$\Gamma_{i}^{x} \quad$ risk level of input parameters for constraint $\mathrm{i}$

$\Gamma_{r}^{y} \quad$ risk level of output parameters for constraint r

$\hat{x}_{i j} \quad$ maximum deviation from $x_{i j}$

$\hat{y}_{r j} \quad$ maximum deviation from $y_{r j}$

Variables

$\theta_{o} \quad$ Optimal efficiency value for $\mathrm{SC}_{\mathrm{o}}$

$\lambda_{j} \quad$ Efficiency of $\mathrm{SC}_{\mathrm{j}}$

$z_{i}^{x}, p_{i j}^{x}, y_{j}^{x} \quad$ Dual variables for input constraints in robust modeling, proof [1].

$z_{r}^{y}, p_{r j}^{y}, y_{j}^{y} \quad$ Dual variables for output constraints in robust modeling, proof [1].

\section{Robust optimization construction for DEA model}

As we know, DEA models ranks alternatives based on some criteria. We assume each supply chain as a DMU (Decision Making Unit) and with respect to inputs and outputs of each SC we will decide which SC (Supply Chain) is more efficient than the others. For better determining outputs and inputs, we should use a SC performance index system. There are lots of different studies about SC performance index system like Lummus and Vokurka (1999) who used a 4 index system and PRTM (2000); an authoritative supply chain research organization; put 11 indexes in SCOR (Supply Chain Operations Reference) model. Regarding to above studies and rules of DEA the SC performance index system which will be used here has been shown in Table 1.

Table 1

Input-output evaluation index system for each SC

\begin{tabular}{lllc}
\hline Factors & First index system & Name of index & Unit of index \\
\hline \multirow{4}{*}{ Input } & \multirow{2}{*}{ Cost } & Direct costs & $\$$ \\
& & Operation costs & $\$$ \\
& \multirow{2}{*}{ Time } & Transaction expenses & $\$$ \\
& HR & Order lead time & Day \\
\cline { 2 - 4 } & \multirow{2}{*}{ Flexibility } & Total volume of employees & Person \\
\cline { 2 - 4 } Output & \multirow{2}{*}{ Financial } & Product flexibility & No dimension \\
& \multirow{2}{*}{ Service } & Delivery flexibility & $1 /$ day \\
& \multirow{2}{*}{ Seles volume } & $\$$ \\
& & Net profit & $\$$ \\
\hline
\end{tabular}

In this paper, we use CCR DEA model and changed it to a robust model. 
$\min \theta_{o}$

subject to

$$
\begin{array}{ll}
\sum_{j} x_{i j} \lambda_{j} \leq \theta_{o} x_{i o} & \forall i \\
\sum_{j} y_{r j} \lambda_{j} \leq y_{r o} & \forall r \\
\lambda_{j} \geq 0 &
\end{array}
$$

In model (4), $X_{i j}$ is the parameter of input $\mathrm{i}$ for $\mathrm{SC}_{\mathrm{j}}$, and $Y_{r j}$ is the parameter of output $\mathrm{r}$ for $\mathrm{SC} \mathrm{j}$.

\section{Robust counterpart model for CCR of DEA models}

$$
\operatorname{Min} \boldsymbol{\theta}_{\mathrm{o}}
$$

subject to

$$
\begin{array}{ll}
\sum_{j} x_{i j} \lambda_{j}+z_{i}^{x} \cdot \Gamma_{\mathrm{i}}^{\mathrm{x}}+\sum_{j \epsilon J_{i}} p_{i j}^{x} \cdot x_{i j} \leq \theta_{o} \cdot x_{i o} & \forall i \\
\sum_{j} y_{r j} \lambda_{j}+z_{r}^{y} \cdot \Gamma_{\mathrm{r}}^{\mathrm{y}}+\sum_{j \in J r} p_{r j}^{y} \leq y_{r o} & \forall r \\
z_{i}^{x}+p_{i j}^{x} \geq \hat{x}_{i j} \cdot y_{j}^{x} & \forall(i, j) \epsilon J_{i} \\
z_{r}^{y}+p_{r j}^{y} \geq \hat{y}_{r j} \cdot y_{j}^{y} & \forall(r, j) \epsilon J_{r} \\
-y_{j}^{x} \leq \lambda_{j} \leq y_{j}^{x} & \forall j \\
-y_{j}^{y} \leq \lambda_{j} \leq y_{j}^{y} & \forall j \\
p_{i j}^{x} \geq 0 & \forall(i, j) \epsilon J_{i} \\
p_{r j}^{y} \geq 0 & \forall(r, j) \epsilon J_{r} \\
y_{j}^{x}, y_{j}^{y}, \lambda_{j} \geq 0 & \forall j \\
z_{i}^{x} \geq 0 & \forall i \\
z_{r}^{y} \geq 0 & \forall r
\end{array}
$$

In constraints (6) and (7) the terms $z_{i}^{x} \cdot \Gamma_{\mathrm{i}}^{\mathrm{x}}$ and $z_{r}^{y}$. $\Gamma_{\mathrm{r}}^{\mathrm{y}}$ indicate risk levels and the terms $\sum_{j \in J_{i}} p_{i j}^{x} \cdot x_{i j}$ and $\sum_{j \epsilon J r} p_{r j}^{y}$ indicate dual values of coefficients which addressed as uncertainty. Eqs. (8) to (16) are dual constraints. The proof is in given by Sim (2004), pages 1 to 28.

\section{Numerical example}

For efficiency evaluation of our proposed model, we have solved some numerical examples. Here is an example of a supply chain network from oil facility supporting industry, which includes 10 supply chains as subsystems. Each supply chain has some inputs and outputs. Our purpose is to evaluate the performance of these supply chains under uncertain environment terms to determine which of them are more efficient in different situations and rank these suppliers regarding to these uncertain conditions. First, we model the supply chain network for both deterministic CCR model and Robust DEA model under nominal data, the results are indicated in Table 3 and Input and output Nominal data of ten supply chains from oil facility supporting industry are shown in Table 2. Then for each value of $\Gamma$ (Risk level or deviation value of nominal data), we solve problem under real data by using; $\left[\bar{a}_{\mathrm{ij}}-\hat{a}_{\mathrm{ij}}, \bar{a}_{\mathrm{ij}}+\hat{a}_{\mathrm{ij}}\right]$; interval and generating real data for each uncertain $a_{i j}$. As mentioned in introduction part, nominal data $\bar{a}_{\mathrm{ij}}$ and its interval $\hat{a}_{\mathrm{ij}}$ are taken from an oil facility supporting industry. 
Table 2

Nominal data for inputs-outputs

\begin{tabular}{llll}
\hline Parameter & Corresponding random distribution & Parameter & Corresponding random distribution \\
\hline $\mathrm{x}_{1 \mathrm{j}}$ & Uniform $(1600,2200)$ & $\mathrm{y}_{1 \mathrm{j}}$ & Uniform $(1,10)$ \\
$\mathrm{x}_{2 \mathrm{j}}$ & Uniform $(500,700)$ & $\mathrm{y}_{2 \mathrm{j}}$ & Uniform $(5000,8000)$ \\
$\mathrm{X}_{3 \mathrm{j}}$ & Uniform $(800,1000)$ & $\mathrm{y}_{3 \mathrm{j}}$ & Uniform $(1500,2300)$ \\
$\mathrm{x}_{4 \mathrm{j}}$ & Uniform $(20,40)$ & & \\
\hline
\end{tabular}

In Table 2: $\mathrm{X}_{1 \mathrm{j}}$ denotes direct costs, $\mathrm{X}_{2 \mathrm{j}}$ denotes operational costs, $\mathrm{X}_{3 \mathrm{j}}$ shows transaction expenses, $\mathrm{X}_{4 \mathrm{j}}$ denotes order lead time, $\mathrm{Y}_{1 \mathrm{j}}$ denotes product flexibility, $\mathrm{Y}_{2 \mathrm{j}}$ is delivery flexibility and $\mathrm{Y}_{3 \mathrm{j}}$ shows net profit. We used the constant value for $\Gamma_{\mathrm{i}}$ to get more realistic results, such that if $\Gamma_{\mathrm{i}}=4$ then from 10 coefficient of each constraint, just 4 coefficient are uncertain, and when $\Gamma_{\mathrm{i}}=10$, it means all coefficients of each constraint are uncertain .It should be considered that our proposed model indicates performance of supply chains in more real situations by varying parameters and determining which arrangement of supply chains will be more appropriate and efficient in supply chain network.

Table 3

DMUs efficiency under nominal data

\begin{tabular}{lcccccccccccc}
\hline & CCR model & \multicolumn{2}{c}{ Robust model in different level of uncertainty } & & & & & \\
& $\Gamma_{\mathrm{i}}=0$ & $\Gamma_{\mathrm{i}}=1$ & $\Gamma_{\mathrm{i}}=2$ & $\Gamma_{\mathrm{i}}=3$ & $\Gamma_{\mathrm{i}}=4$ & $\Gamma_{\mathrm{i}}=5$ & $\Gamma_{\mathrm{i}}=6$ & $\Gamma_{\mathrm{i}}=7$ & $\Gamma_{\mathrm{i}}=8$ & $\Gamma_{\mathrm{i}}=9$ & $\Gamma_{\mathrm{i}}=10$ \\
\hline dmu01 & 1 & 1 & 1 & 1 & 0.98 & 0.98 & 0.98 & 1 & 1 & 1 \\
dmu02 & 0.33 & 0.34 & 0.34 & 0.34 & 0.34 & 0.34 & 0.34 & 0.34 & 0.34 & 0.34 & 0.34 \\
dmu03 & 1 & 0.99 & 1 & 0.99 & 0.99 & 0.98 & 0.98 & 0.98 & 0.99 & 0.99 & 0.99 \\
dmu04 & 1 & 1 & 1 & 1 & 1 & 1 & 1 & 1 & 1 & 1 & 1 \\
dmu05 & 0.82 & 0.84 & 0.86 & 0.86 & 0.86 & 0.86 & 0.86 & 0.86 & 0.86 & 0.86 & 0.86 \\
dmu06 & 0.56 & 0.58 & 0.58 & 0.58 & 0.58 & 0.58 & 0.58 & 0.58 & 0.58 & 0.58 & 0.58 \\
dmu07 & 1 & 0.96 & 0.96 & 0.95 & 0.95 & 0.96 & 0.95 & 0.96 & 0.96 & 0.96 & 0.96 \\
dmu08 & 1 & 0.98 & 1 & 1 & 1 & 1 & 1 & 1 & 1 & 1 & 1 \\
dmu09 & 1 & 1 & 1 & 1 & 1 & 0.99 & 1 & 1 & 1 & 1 & 1 \\
dmu10 & 0.73 & 0.89 & 0.86 & 0.89 & 0.96 & 0.93 & 0.96 & 0.93 & 0.93 & 0.93 & 0.86 \\
\hline
\end{tabular}

\section{Comparison between CCR and ROBUST DEA model}

In this part, we compare the performance between CCR and ROBUST DEA model. In the rest of the section, $\left(\Gamma_{\mathrm{i}}=0\right)$ represents CCR model which in table number 3, 4, 5 CCR model specified in a particular column and in Table 7, first row i.e. $\left(\Gamma_{\mathrm{i}}=0\right)$ represents existing CCR model. After modeling and solving several problems in uniform data mentioned in Table 2, by using OR software, we have DMUs ranking (descending) based on CCR model $\left(\Gamma_{\mathrm{i}}=0\right)$ regarding to Table 3 such as:

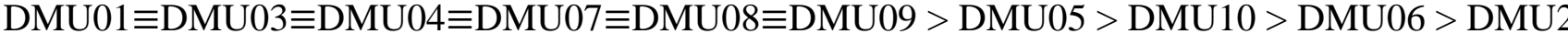

It means the performance of SC number 1,3,4,7,8 and 9 is equal. It is perceived that ranking of DMUs changes by varying $\Gamma_{\mathrm{i}}$. In Table 3 for each $\Gamma_{\mathrm{i}}=\mathrm{j}(\mathrm{j}=0,1, \ldots, 10)$, we generate data in range of $\left[\bar{a}_{\mathrm{ij}}-\hat{a}_{\mathrm{ij}}\right.$, $\bar{a}_{\mathrm{ij}}+\hat{a}_{\mathrm{ij}}$ ], 20 times randomly. In Table 4, the mean of efficiency of each SC (DMU) under every $\Gamma_{\mathrm{i}}=\mathrm{j}$ $(j=0,1, \ldots, 10)$ for twenty time realizations have been computed, then in Table 5 these efficiencies have been sorted in non-increasing order. In Table 5 , for better understanding we resort Table 5 regarding to DMU's performance for each level of uncertainty $\Gamma_{\mathrm{i}}=\mathrm{j}(\mathrm{j}=0,1, \ldots, 10)$.

\section{Table 4}

Mean efficiency of SCs based on real data

\begin{tabular}{|c|c|c|c|c|c|c|c|c|c|c|c|}
\hline & $\Gamma_{\mathrm{i}}=0$ & $\Gamma_{\mathrm{i}}=1$ & $\Gamma_{\mathrm{i}}=2$ & $\Gamma_{\mathrm{i}}=3$ & $\Gamma_{\mathrm{i}}=4$ & $\Gamma_{\mathrm{i}}=5$ & $\Gamma_{\mathrm{i}}=6$ & $\Gamma_{\mathrm{i}}=7$ & $\Gamma_{\mathrm{i}}=8$ & $\Gamma_{\mathrm{i}}=9$ & $\Gamma_{\mathrm{i}}=10$ \\
\hline dmu01 & 0.768 & 0.786 & 0.798 & 0.85 & 0.848 & 0.848 & 0.846 & 0.844 & 0.836 & 0.836 & 0.848 \\
\hline dmu02 & 1 & 0.878 & 0.924 & 0.94 & 0.952 & 0.95 & 0.948 & 0.948 & 0.948 & 0.95 & 0.948 \\
\hline dmu03 & 0.906 & 0.93 & 0.952 & 0.952 & 0.944 & 0.944 & 0.942 & 0.946 & 0.946 & 0.944 & 0.942 \\
\hline dmu04 & 0.96 & 0.964 & 0.964 & 0.96 & 0.97 & 0.972 & 0.964 & 0.972 & 0.972 & 0.958 & 0.966 \\
\hline dmu05 & 0.944 & 0.918 & 0.924 & 0.956 & 0.952 & 0.952 & 0.952 & 0.952 & 0.952 & 0.946 & 0.952 \\
\hline dmu06 & 0.926 & 0.858 & 0.85 & 0.858 & 0.844 & 0.844 & 0.844 & 0.844 & 0.844 & 0.84 & 0.844 \\
\hline dmu07 & 1 & 0.936 & 0.974 & 0.976 & 0.984 & 0.984 & 0.982 & 0.984 & 0.982 & 0.982 & 0.984 \\
\hline dmu08 & 0.772 & 0.762 & 0.784 & 0.792 & 0.788 & 0.79 & 0.768 & 0.788 & 0.786 & 0.788 & 0.788 \\
\hline dmu09 & 0.936 & 0.94 & 0.948 & 0.95 & 0.95 & 0.952 & 0.94 & 0.948 & 0.952 & 0.952 & 0.948 \\
\hline dmu10 & 0.898 & 0.878 & 0.876 & 0.896 & 0.896 & 0.896 & 0.892 & 0.896 & 0.896 & 0.894 & 0.896 \\
\hline
\end{tabular}




\section{Table 5}

Mean ordered efficiency of SCs in table 5 for each $\Gamma_{\mathrm{i}}=\mathrm{j}(\mathrm{j}=0,1, \ldots, 10)$

\begin{tabular}{lllllllllllll}
\hline & $\Gamma_{\mathrm{i}}=0$ & $\Gamma_{\mathrm{i}}=1$ & $\Gamma_{\mathrm{i}}=2$ & $\Gamma_{\mathrm{i}}=3$ & $\Gamma_{\mathrm{i}}=4$ & $\Gamma_{\mathrm{i}}=5$ & $\Gamma_{\mathrm{i}}=6$ & $\Gamma_{\mathrm{i}}=7$ & $\Gamma_{\mathrm{i}}=8$ & $\Gamma_{\mathrm{i}}=9$ & $\Gamma_{\mathrm{i}}=10$ \\
\hline \multirow{6}{*}{$\mathrm{DMU}_{\mathrm{i}}$} & 1 & 0.964 & 0.974 & 0.976 & 0.984 & 0.984 & 0.982 & 0.984 & 0.982 & 0.982 & 0.984 \\
& 1 & 0.94 & 0.964 & 0.96 & 0.97 & 0.972 & 0.964 & 0.972 & 0.972 & 0.958 & 0.966 \\
& 0.96 & 0.936 & 0.952 & 0.956 & 0.952 & 0.952 & 0.952 & 0.952 & 0.952 & 0.952 & 0.952 \\
& 0.944 & 0.93 & 0.948 & 0.952 & 0.952 & 0.952 & 0.948 & 0.948 & 0.952 & 0.95 & 0.948 \\
& 0.936 & 0.918 & 0.924 & 0.95 & 0.95 & 0.95 & 0.942 & 0.948 & 0.948 & 0.946 & 0.948 \\
& 0.926 & 0.878 & 0.924 & 0.94 & 0.944 & 0.944 & 0.94 & 0.946 & 0.946 & 0.944 & 0.942 \\
& 0.906 & 0.878 & 0.876 & 0.896 & 0.896 & 0.896 & 0.892 & 0.896 & 0.896 & 0.894 & 0.896 \\
& 0.898 & 0.858 & 0.85 & 0.858 & 0.848 & 0.848 & 0.846 & 0.844 & 0.844 & 0.84 & 0.848 \\
& 0.772 & 0.786 & 0.798 & 0.85 & 0.844 & 0.844 & 0.844 & 0.844 & 0.836 & 0.836 & 0.844 \\
& 0.768 & 0.762 & 0.784 & 0.792 & 0.788 & 0.79 & 0.768 & 0.788 & 0.786 & 0.788 & 0.788 \\
\hline
\end{tabular}

\section{Table 6}

Final ranking of SCs efficiency based on real data

\begin{tabular}{|c|c|c|c|c|c|c|c|c|c|c|}
\hline$\Gamma_{\mathrm{i}}$ & Rank1 & Rank2 & Rank3 & Rank4 & Rank5 & Rank6 & Rank7 & Rank8 & Rank9 & Rank10 \\
\hline 0 & 7,2 & 4 & 5 & 9 & 6 & 3 & 10 & 8 & 1 & \\
\hline 1 & 4 & 9 & 7 & 3 & 5 & 2,10 & 6 & 1 & 8 & \\
\hline 2 & 7 & 4 & 3 & 9 & 5,2 & 10 & 6 & 1 & 8 & \\
\hline 3 & 7 & 4 & 5 & 3 & 9 & 2 & 10 & 6 & 1 & 8 \\
\hline 4 & 7 & 4 & 2,5 & 9 & 3 & 10 & 1 & 6 & 8 & \\
\hline 5 & 7 & 4 & 5,2 & 9 & 3 & 10 & 1 & 6 & 8 & \\
\hline 6 & 7 & 4 & 5 & 2 & 3 & 9 & 10 & 1 & 6 & 8 \\
\hline 7 & 7 & 4 & 5 & 2,9 & 3 & 10 & 6,1 & 8 & & \\
\hline 8 & 7 & 4 & 5,9 & 2 & 3 & 10 & 6 & 1 & 8 & \\
\hline 9 & 7 & 4 & 9 & 2 & 5 & 3 & 10 & 6 & 1 & 8 \\
\hline 10 & 7 & 4 & 5 & 2,9 & 3 & 10 & 1 & 6 & 8 & \\
\hline
\end{tabular}

In Table 4, uncertain parameters in each constraint have been determined by $\Gamma_{\mathrm{i}}$ that could be in $[0,10]$. In Table 6, each cell denotes the number of supply chain(s) in a particular rank and level. As it's obvious in Table 6, by increasing uncertainty level (increasing $\Gamma_{\mathrm{i}}$ ); i.e. increasing number of uncertain parameters; the final ranking of supply chains efficiency goes to:

DMU07 $>$ DMU04 $>$ DMU05 $>$ DMU09 $>$ DMU02 $>$ DMU03 $>$ DMU10 $>$ DMU06 $>$ DMU01 $>$ DMU08

This result will be shown in Fig. 2:

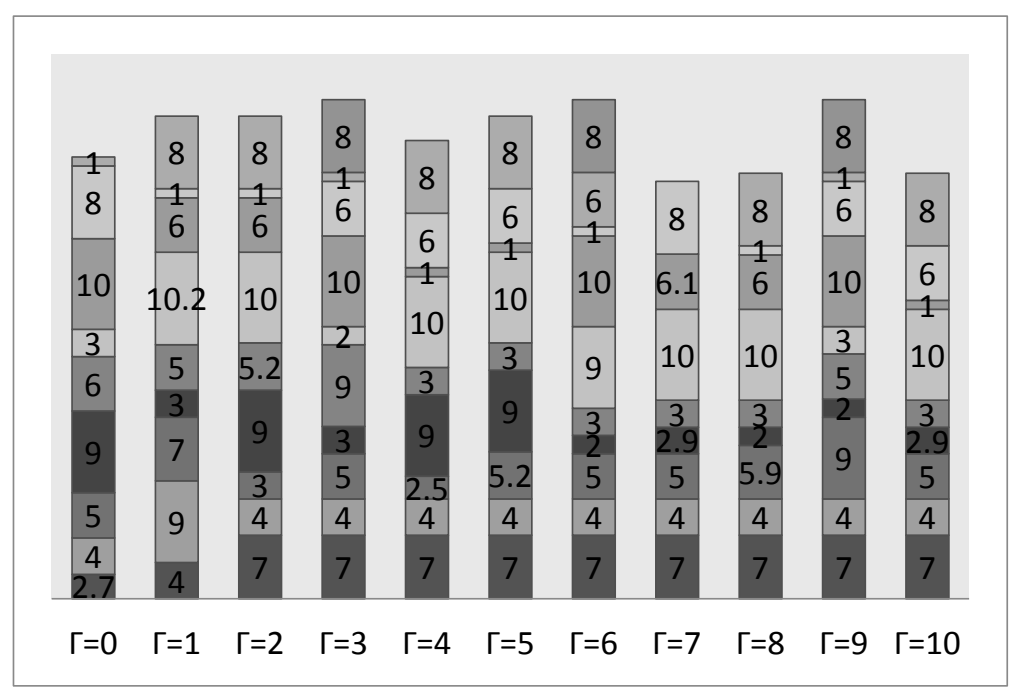

Fig. 2. Supply chain ranking in different level of uncertainty

Meanwhile, it should be considered that in each level of uncertainty of Table 6, we are able to determine the best ranking of DMUs. For example, if $\Gamma_{\mathrm{i}}=5$, i.e. there are 5 uncertain parameters in each constraint, then ranking of DMUs would be: 
DMU07 > DMU04 > DMU02 > DMU05 > DMU09 > DMU03 > DMU10 > DMU01 > DMU06 > DMU08

\section{Conclusions}

This paper has presented a robust data envelopment analysis model to evaluate supply chain performance. In this paper, we had a supply chain network where its data came from an oil facility supporting industry. In every SC, there are some input-output parameters, which have been considered under uncertainty. As a DEA model, every supply chain is assumed like a Decision Making Unit (DMU). We have applied a SC performance index system to determine input-output parameters indicated. Our proposed model not only determined the appropriate rank of DMUs in each environmental under uncertainty level, but also was able to offer a robust ranking of DMUs which was capable of providing a suitable performance in any different level of uncertainties. As a comparison, there is a ranking of SC's efficiency based on real world data given in Table 6, the first row i.e. $\left(\Gamma_{\mathrm{i}}=0\right)$ shows the inflexible CCR DEA model ranking of DMUs on any level of uncertainty, but our robust model gives us different ranking in any level of uncertainty. Obviously, the result of this research will be useful in strategic decision making in SCN (Supply Chain Network) for determining the most efficient suppliers. The considered problem is in initial stage of investigation and future researches can be accomplished based on the results of this paper, such as combining the other robust optimization approaches whit classical DEA models, especially for non-linear DEA models. Also some new DEA models like Rough DEA model could be integrated with robust optimization approach to evaluate DMUs with uncertain parameters.

\section{Acknowledgements}

This article has been supported by grant no. 4/2957 from Kharazmi University, Tehran ,Iran and authors thank research department of university for their good supports during this study.

\section{References}

Adida, E., \& Perakis, G. (2006). A robust optimization approach to dynamic pricing and inventory control with no backorders. Mathematical Programing, 107(1), 97-129.

Amin, S.H., \& Zhang, G. (2012). A proposed mathematical model for closed-loop network configuration based on product life cycle. International Journal of Advanced Manufacturing Technology, 58(5-8), 791-801.

Azoulay-Schwartz, R., Kraus, S., \& Wilkenfeld, J. (2004). Exploitation vs. exploration: choosing a supplier in an environment of incomplete information. Decision Support System, 38(1), 1-18.

Barbarosoglu, G., \& Yazgac, T. (1997). An application of the analytic hierarchy process to the supplier selection problem. Journal of Production Innovation Management, 38 (1), 14-21.

Ben-Tal, A., \&Nemirovski, A. (2000).Robust solutions of linear programming problems contaminated with uncertain data. Mathematical Programming, 88(3), 411-424.

Ben-Tal,A., \& Nemirovski, A. (1998). Robust convex optimization. Journal of Mathematical Operations Research, 23(4), 769-805.

Cebi, F., \& Bayraktar, D. (2003). An integrated approach for supplier selection. Logistics Information Management, 16(6), 395-400.

Chan, F.T.S. (2003). Performance measurement in a supply chain .International Journal of Advanced Manufacturing Technology, 21 (7), 534-548.

Chiang, T.A, \& Che, Z.H. (2010). A fuzzy robust evaluation model for selecting and ranking NPD projects using Bayesian belief network and weight-restricted DEA. Expert Systems with Applications, 37(11), 7408-7418.

Chow, G., Heaver, T.D., \& Henriksson, L.E. (1994). Logistics performance: definition and measurement. International Journal of Physical Distribution, 24(1), 17-28.

Easton, L., Murphy, D., \& Pearson, N.J. (2002). Purchasing performance evaluation: with data envelopment analysis. European Journal of Purchasing \& Supply Management, 8(3),123-134. 
El-Ghaoui, L., Oustry, F., \& Lebret, H. (1998). Robust solutions to uncertain semidefinite programs. SIAM Journal of Optimization, 9(1), 33-52.

Gabrel, V., Murat, C., \& Thiele, A. (2012). Recent advances in robust optimization and robustness: An overview, http://www.optimization-online.org.

Jahanshahloo, G.R., Pourkarimi, L., \& Zarepishe, M. (2006). Modified MAJ model for ranking decision making units in data envelopment analysis. Applied Mathematical Computation, 174(2), 1054-1059.

Khezrimotlagh, D., Salleh,S., \& Mohsenpour, Z. (2013). A new robust mixed integer-valued model in DEA. Applied Mathematical Modelling, 37(24), 1-13.

Kleinsorge, K.I. (1992). Data envelopment analysis for monitoring customer-supplier relationships. Journal of Accounting and Public Policy, 11(4), 357-372.

Kumar, M., Vrat, P., \& Shankar, R. (2004). A Fuzzy goal programming approach for vendor selection problem in a supply chain. Computers and Industrial Engineering, 46 (1), 69-85.

Lummus, R., \& Vokurka, R. (1999). Defining supply chain management: A historical perspective and practical guide line. Industrial Management and Data System, 99(1), 11-17.

Mahmoudzadeh, M. Sadjadi, S.J., \& Mansour, S. (2013).Robust optimal dynamic production/pricing policies in a closed-loop system. Applied Mathematical Modelling, 37(16-17), 8141-8161.

Narasimhan, R., Tallurai, S., \& Mendez, D. (2001). Supplier evaluation and rationalization via data envelopment analysis: an empirical examination. Supply Chain Management, 37 (3), 28-37.

Ohdar, R., \& Ray, P.K. (2004). Performance measurement and evaluation of suppliers in supply chain: an evolutionary fuzzy-based approach. Journal of Manufacturing Technology, 15 (8), 723-734.

Quariguasi FrotaNeto, J. (2007). A methodology for assessing eco-efficiency in logistics networks. European Journal of Operational Research, 193(3), 670-682.

Ross, A., \& Droge, C. (2002). An integrated benchmarking approach to distribution center performance using DEA modeling. Journal of Operations Management, 20(1), 19-32.

Soltani, R., \& Sadjadi, S.J. (2010). Scheduling trucks in cross-docking systems: A robust metaheuristics approach. Transportation Research Part E, 46(5), 650-666.

Sadjadi, S.J., \& Omrani, H. (2010). A bootstrapped robust data envelopment analysis model for efficiency estimating of telecommunication companies in Iran. Telecommunications Policy, 34(4), 221-232.

Sadjadi , S.J. , Omrani , H. , Abdollahzadeh , S. , Alinaghian , M. ,\& Mohammadi, H.(2011). A robust super-efficiency data envelopment analysis model for ranking of provincial gas companies in Iran. Expert Systems with Applications, 38(9), 10875-10881.

Sadjadi, S.J., \& Omrani, H. (2008). Data envelopment analysis with uncertain data: An application for Iranian electricity distribution companies. Energy Policy, 36(11), 4247-4254.

Sadjadi, S.J., Omrani, H., Makui, A., \& Shahanaghi, K. (2011). An interactive robust data envelopment analysis model for determining alternative targets in Iranian electricity distribution companies. Expert Systems with Applications, 38(8), 9830-9839.

Sadjadi,S.J ,Gharakhani,M.,\& Safari,E.(2012). Robust optimization framework for cardinality constrained portfolio problem. Applied Soft Computing, 12(1), 91-99.

Sim, M. (2004). Robust Optimization. Ph.D. Dissertation, Massachusetts Institute of Technology, 1540.

Sinuany-Stern, Z., Mehrez, A., \& Hadad, Y. (2000). An AHP/DEA methodology for ranking decision making units. International Transactions in Operational Research, 7(2), 109-124.

Talluria, S., Narasimhan, R., \& Anand, N. (2006). Vendor performance with supply risk: a chancedconstrained DEA approach. International Journal of Production Economics, 100(2), 212-222.

Weber, A.C., \& Desai, A. (1998). Non-cooperative negotiation strategies for vendor selection. European Journal of Operational Research, 108(1), 208-223.

Wu, D., Xu, J., \& Li, B. (2009). Rough data envelopment analysis and its application to supply chain performance evaluation. International Journal of Production Economics, 122(2), 628-638.

Wu, D., \& Olson, L.D. (2008). A comparison of stochastic dominance and stochastic DEA for vendor evaluation. International Journal of Production Research, 46(8), 2313-2327. 